SEQUENTIAL METHODS IN STATISTICS

BANACH CENTER PUBLICATIONS, VOLUME 16

PWN $\cdots$ POLISH SCIENTIFIC PUBLISHERS

WARSAW 1985

\title{
A MODIFICATION OF SUDAKOV'S LEMMA AND EFFICIENT SEQUENTIAL PLANS FOR SOME JUMP MARKOV PROCESSES
}

\author{
R. MAGIERA and R. RÓŻAŃSK I \\ Institute of Mathematics, Wroclaw Technical University, \\ Wroclaw, Poland
}

\section{A modification of Sudakov's lemma and the Cramér-Rao inequality}

In $1968 \mathrm{~S}$. Trybula proved in [7] that for the Poisson process $\xi(t), t \geqslant 0$, with the parameter $\lambda$ the joint distribution $m_{\lambda}$ of a random vector $(\tau, \xi(\tau))$, where $\tau$ is a Markov stopping time, is absolutely continuous with respect to $m_{\lambda_{0}}$ for some $\lambda_{0}$ with the density $d m_{\lambda} / d m_{\lambda_{0}}$. Using this fact, the author obtained a characterization of efficient sequential plans for the Poisson process with an unknown parameter $\lambda$. In the same article analogous results for the negative-binomial, gamma and Wiener processes were obtained. Later, in 1969, V. N. Sudakov ([6]) proved the following lemma, generalizing the result obtained by Trybuła.

Lemma ([6] or [2], 55-59). Let $\left(\Omega, \mathscr{F}, P_{\theta}\right)$ be a probability space and let $\xi(t), t \geqslant 0$, be a stochastic process with right-continuous realizations $-P_{9}$ almost surely for every $\vartheta \in \Theta$.

We assume that $\xi(t)$ is a sufficient statistic for $\vartheta$ and the one-dimensional distribution $P_{\vartheta}(\xi(t) \in B)$ of $\xi(t), t \geqslant 0$, is absolutely continuous with respect to the one-dimensional distribution $P_{\vartheta_{0}}(\xi(t) \in B)$ and the density function $h\left(t, x ; 9, \vartheta_{0}\right)$ is continuous.

Then for any Markov stopping time $\tau$ the joint distribution $m_{\vartheta}$ of $(\tau, \xi(\tau))$ is absolutely continuous with respect to the joint distribution $m_{\vartheta_{0}}$ and

$$
\begin{gathered}
\frac{d m_{\vartheta}}{d m_{\vartheta_{0}}}(u)=h\left(t(u), x(u) ; \vartheta, \vartheta_{0}\right), \\
u=(t(u), x(u)) \in U=[0, \infty) \times R^{\prime} .
\end{gathered}
$$

Let us introduce the following notation:

$D([0, \infty))$ - the space of functions $x(\cdot):[0, \infty) \rightarrow R^{k}$ which are right continuous and have left-side limits. 
$\mathscr{Z}$ - the least $\sigma$-algebra of subsets of $D([0, \infty))$ with respect to which the coordinate mappings $x(t): D([0, \infty)) \rightarrow R^{k}$ are measurable.

$\mathscr{D}_{t}$ - the least $\sigma$-algebra of subsets of $D([0, \infty))$ with respect to which the coordinate mappings $x(s): D([0, \infty)) \rightarrow R^{k}, s \in[0, t]$ are measurable.

$\mu_{9}$ - a probability measure defined on $(D([0, \infty))$, $\mathscr{D})$, depending on the parameter $\vartheta=\left(\vartheta_{1}, \vartheta_{2}, \ldots, \vartheta_{s}\right) \in \Theta \subset R^{s}$.

Definition 1. A Markov stopping time is a random variable $\tau$ : $D([0, \infty)) \rightarrow[0, \infty]$ for which

$$
\{x(\cdot): \tau(x(\cdot)) \leqslant t\} \in \mathscr{D}_{t} \quad \text { for every } \quad t \geqslant 0 .
$$

Further we also assume that

$$
\mu_{3}(\{x(\cdot): \tau(x(\cdot))<\infty\})=1 \quad \text { for every } \vartheta \in \Theta .
$$

$1^{\circ}$. For every $t$, let $Z(t, x(\cdot))$, be the mapping from $D([0, \infty))$ to $R^{l}$, measurable with respect to $\mathscr{C}_{1}$ and right-continuous with respect to $t-\mu_{3}$ almost surely for every $\vartheta \in \Theta$.

$2^{0}$ By $\mu_{9, t}$ we denote the restriction of $\mu_{9}$ to the $\sigma$-algebra $\mathscr{D}_{1}$. Let us assume that $\mu_{9,1}$ is absolutely continuous with respect to $\mu_{9_{0}, 4}$ and

$$
\frac{d \mu_{\vartheta_{1} t}}{d \mu_{\vartheta_{0}, t}}(x(\cdot))=h\left(t, Z(t, x(\cdot)) ; \vartheta, \vartheta_{0}\right),
$$

where $h$ is a continuous function and $Z(t)=Z(t, x(\cdot))$ is a mapping satisfying the previous condition.

The problem is what we can say about the joint distribution of the random vector $(\tau, Z(\tau))$.

$3^{\circ}$. Let $U=[0, \infty) \times R^{l}=T \times R^{l}$,

$$
U \in u=(t(u), z(u)) .
$$

$\mathscr{B}_{U}$ - the $\sigma$-algebra of Borel subsets of $U$.

On $\left(U, \mathscr{B}_{U}\right)$ we define, for every $A \in \mathscr{B}_{U}$, the measure $m_{3}$ generated by the statistics $Z$ and the Markov time $\tau$ :

$$
m_{\vartheta}(A)=\mu_{\vartheta}(\{x(\cdot):(\tau(x(\cdot)), Z(\tau(x(\cdot)), x(\cdot))) \in A\}) .
$$

LEMMA 1 ([4]). Under assumptions $1^{\circ}-3^{\circ}$ the measure $m_{3}$ is absolutely continuous with respect to the measure $m_{9_{0}}$ and the density function takes the form:

$$
\frac{d m_{9}}{d m_{\vartheta_{0}}}(u)=h\left(t(u), z(u) ; \vartheta, \vartheta_{0}\right) .
$$

Definition 2. By a sequential plan we mean the pair $(\tau, f(\tau, Z(\tau)))$, where $\tau$ is a Markov stopping time and $f: U \rightarrow R$ is a $\mathscr{B}_{U}$-measurable function. 
The function $f$ will be called an estimator of a given function $g(\vartheta)$. We also assume that:

(i) $E_{m_{g}} f=E_{\vartheta} f(\tau, Z(\tau))=g(\vartheta)$,

(ii) $E_{m_{\vartheta}} f^{2}=\int_{U} f^{2}(u) h\left(u ; \vartheta, \vartheta_{0}\right) d m_{\vartheta_{0}}(u)<\infty$ for every $\vartheta \in \Theta$,

(iii) the function $g(9)$ is differentiable and $g(9) \not \equiv$ const. Let us denote:

$$
\begin{aligned}
& V_{\vartheta}(u)=\left(\frac{\partial}{\partial \vartheta_{1}} \log h\left(u ; \vartheta, \vartheta_{0}\right), \ldots, \frac{\partial}{\partial \vartheta_{s}} \log h\left(u ; \vartheta, \vartheta_{0}\right)\right), \\
& \nabla_{\vartheta} g=\left(\frac{\partial}{\partial \vartheta_{1}} g(\vartheta), \ldots, \frac{\partial}{\partial \vartheta_{s}} g(\vartheta)\right), \quad J(\vartheta)=E_{m_{\vartheta}} V_{\vartheta}^{T} V_{\vartheta},
\end{aligned}
$$

$D_{\vartheta}(\cdot)$ - the variance evaluated at $\vartheta$.

We can formulate the following theorem:

THEOREM 1. If a sequential plan $(\tau, f)$ satisfies the regularity conditions which guarantee that

$$
\begin{gathered}
\frac{\partial}{\partial \vartheta_{i}} \int_{U} h\left(u ; \vartheta, \vartheta_{0}\right) d m_{s_{0}}(u)=\int_{U} \frac{\partial}{\partial \vartheta_{i}} h\left(u ; \vartheta, \vartheta_{0}\right) d m_{\vartheta_{0}}(u), \\
\frac{\partial}{\partial \vartheta_{i}} \int_{U} f(u) h\left(u ; \vartheta, \vartheta_{0}\right) d m_{\vartheta_{0}}(u)=\int_{U} f(u) \frac{\partial}{\partial \vartheta_{i}} \log h\left(u ; \vartheta, \vartheta_{0}\right) d m_{\vartheta_{0}}(u),
\end{gathered}
$$

then

$$
D_{\vartheta} f(\tau, Z(\tau)) \geqslant\left(\nabla_{\vartheta} g\right) J^{-1}(\vartheta)\left(\nabla_{\vartheta} g\right)^{T} .
$$

The equality holds at a particular value of $\vartheta$ if and only if

$$
f(u)=\left(\nabla_{\vartheta} g\right) J^{-1}(\vartheta) V_{\Im}^{T}+g(\vartheta) \quad m_{\vartheta_{0}} \text {-almost surely. }
$$

Definition 3. A sequential estimation plan $(\tau, f)$ for $g(\vartheta)$ is said to be efficient at $\vartheta$ if (1) becomes an equality at $\vartheta$.

The estimator $f$ is then called efficient at the value $\vartheta$ and the function $g(\vartheta)$ is efficiently estimable at $\vartheta$.

Definition 4. A sequential estimation plan $(\tau, f)$ for $g(\vartheta)$ is said to be efficient if it is efficient at each $\vartheta \in \Theta$.

The estimator $f$ is then called efficient and the function $g(\vartheta)$ is efficiently estimable. 


\section{The characterization of efficient sequential plans for an $m$-state Markov homogeneous process ([8]).}

Let $\xi=\xi(t), t \geqslant 0$, be a homogeneous $m$-state Markov process with intensity matrix $\Lambda$ and with intensities $\lambda_{i j}$ for $i, j=1,2, \ldots, m(i \neq j)$.

Let $P\left(X_{0}=k\right)=p_{k}, 0<p_{k}<1$ for $k=1,2, \ldots, m ; \sum_{k=1}^{m} p_{k}=1$. Denote $p$ $=\left(p_{1}, \ldots, p_{m}\right), \lambda_{i}=\sum_{\substack{j=1 \\ j \neq i}}^{m} \lambda_{i j}$. Let $N_{i j}(t), i, j=1,2, \ldots, m(i \neq j)$, be the number

of jumps of the process $\xi$ from state $i$ to state $j$ in the interval $[0, t]$, let $T_{i}(t)$, $i=1,2, \ldots, m$, be total time during which the process $\xi$ remains in state $i$ in $[0, t]$, and let $V_{k}, k=1,2, \ldots, m$, be a random variable defined in the following way:

$$
V_{k}= \begin{cases}1 & \text { if } \quad X_{0}=k \\ 0 & \text { otherwise }\end{cases}
$$

Denote

$$
N=\left[\begin{array}{lllll}
0 & N_{12} & N_{22} & \ldots & N_{1 m} \\
N_{21} & 0 & & \ldots & N_{2 m} \\
\ldots & \ldots & & \ldots & \ldots \\
N_{m 1} & N_{m 2} & \ldots & \ldots & 0
\end{array}\right]
$$

$T=\left(T_{1}, T_{2}, \ldots, T_{m}\right), V=\left(V_{1}, V_{2}, \ldots, V_{m}\right)$. Let $\vartheta=(p, \Lambda)$.

The process $\xi$ generates the measure $\mu_{s}$ in the space $D_{m}([0, \infty)) \subset D([0, \infty))$ of right continuous functions with values $1,2, \ldots, m$. Let $\mathscr{F}$ be the least $\sigma$-algebra of subsets of $D_{m}$ with respect to which the

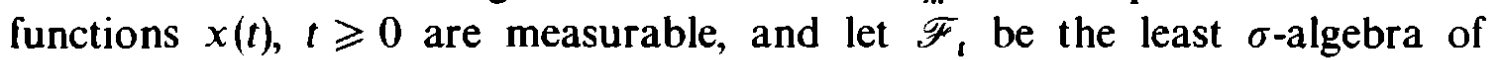
subsets of $D_{m}$ with respect to which $x(s), s \in[0, t]$ are measurable. If $\mu_{9, t}$ denotes the restriction of $\mu_{9}$ on $\mathscr{F}_{t}$, then we have $\mu_{s, t} \ll \mu_{s_{0}, t}$ and

$$
\begin{aligned}
\frac{d \mu_{9, t}}{d \mu_{\vartheta_{0}, t}} & =\prod_{k=1}^{m}\left(\frac{p_{k}}{p_{k}^{0}}\right)^{v_{k}} \prod_{\substack{i, j=1 \\
i \neq j}}^{m}\left(\frac{\lambda_{i j}}{\lambda_{i j}^{0}}\right)^{n_{i j}(t)} \exp \left[\sum_{\substack{r, s=1 \\
r \neq s}}^{m}\left(\lambda_{r s}^{0}-\lambda_{r s}\right) t_{r}(t)\right] \\
& =c\left(v(t), n(t), t(t) ; \vartheta_{0}\right) \prod_{k=1}^{m} p_{k}^{v_{k}} \prod_{\substack{i, j=1 \\
i \neq j}}^{m} \lambda_{i j}^{n_{i j}(t)} \exp \left[-\sum_{\substack{r, s=1 \\
r \neq s}}^{m} \lambda_{r s} t_{r}(t)\right],
\end{aligned}
$$

where $v_{k}, n_{i j}(t), t_{i}(t), v, n(t), t(t)$ are the values of the random variables $V_{k}$, $N_{i j}(t), T_{i}(t), V, N(t), T(t)$, respectively.

Let $\tau$ be a Markov stopping time with respect to $\mathscr{F}_{t}$. By the modification of Sudakov's lemma it follows that the joint distribution $m_{\vartheta}$ of 
$(\tau, V, N(\tau), T(\tau))$ is absolutely continuous with respect to the distribution $m_{\vartheta_{0}}$ for some $\vartheta_{0}$ and

$$
\frac{d m_{3}}{d m_{\vartheta_{0}}}=c\left(v, n, t ; \vartheta_{0}\right) \prod_{k=1}^{m} p_{k}^{v_{k}} \prod_{\substack{i, j=1 \\ i \neq j}}^{m} \lambda_{i j}^{n_{i j}} \exp \left(-\sum_{\substack{r, s=1 \\ r \neq s}}^{m} \lambda_{r s} t_{r}\right),
$$

where $v_{k}, n_{i j}, t_{r}, v, n, t$ are the values of the random variables $V_{k}, N_{i j}(\tau), T_{r}(\tau)$, $V, N(\tau), T(\tau)$, respectively.

$$
\text { Denote } g_{k}^{\prime}=\frac{\partial g}{\partial p_{k}}, g_{i j}^{\prime}=\frac{\partial g}{\partial \lambda_{i j}}
$$

Lemma 2. If $f=f(V, N, T)$ is an estimator of $E f=g(p, \Lambda)$ fulfilling regularity conditions then

$$
\begin{gathered}
E\left[\left(p_{m} V_{k}-p_{k} V_{m}\right) f\right]=p_{k} p_{m} g_{k}^{\prime}, \\
E\left[\left(N_{i j}-\lambda_{i j} T_{i}\right) f\right]=\lambda_{i j} g_{i j}^{\prime}, \\
E\left(p_{m} V_{k}-p_{k} V_{m}\right)^{2}=p_{k} p_{m}\left(p_{k}+p_{m}\right), \\
E\left[\left(p_{m} V_{k}-p_{k} V_{m}\right)\left(p_{m} V_{k^{\prime}}-p_{k^{\prime}} V_{m}\right)\right]=p_{k} p_{k^{\prime}} p_{m}, \\
E\left[\left(N_{i j}-\lambda_{i j} T_{i}\right)\left(N_{i, j}-\lambda_{i, j}, T_{i}\right)\right]=0, \quad(i, j) \neq\left(i^{\prime}, j^{\prime}\right), \\
E\left[\left(p_{m} V_{k}-p_{k} V_{m}\right)\left(N_{i j}-\lambda_{i j} T_{i}\right)\right]=0, \\
E\left(N_{i j}-\lambda_{i j} T_{i}\right)^{2}=\lambda_{i j} E T_{i} .
\end{gathered}
$$

THEOREM 2. For a sequential plan $(\tau, f(\tau, V, N(\tau), T(\tau)))$ satisfying the regularity conditions we have

$$
D_{g} f \geqslant \sum_{k=1}^{m-1} p_{k}\left(g_{k}^{\prime}\right)^{2}-\left(\sum_{k=1}^{m-1} p_{k} g_{k}^{\prime}\right)^{2}+\sum_{\substack{i, j=1 \\ i \neq j}}^{m} \frac{\lambda_{i j}\left(g_{i j}^{\prime}\right)^{2}}{E T_{i}} .
$$

Equality holds at a particular value of $(p, \Lambda)$ if and only if $f$ is a linear function of $w_{k}$ and $w_{i, j}, m_{\vartheta_{0}}$ almost surely, where

$$
w_{k}=\frac{p_{m} v_{k}-p_{k} v_{m}}{p_{k} p_{m}}, \quad w_{i j}=\frac{n_{i j}-\lambda_{i j} t_{i}}{\lambda_{i j}}
$$

So we can formulate the following corollary.

COROLLARY. If $\tau$ is a Markov stopping time, then a nonconstant estimator $f=f(v, n, t)$ is efficient for $E f=g(p, \Lambda)$ at $(p, \Lambda)$ if and only if there exist constants $a_{k}, b_{i j}, i, j=1,2, \ldots, m ; k=1,2, \ldots, m$, not all equal to zero, and $a$ constant $c$ such that

$$
f(v, n, t)=\sum_{k=1}^{m-1} a_{k}\left(p_{m} v_{k}-p_{k} v_{m}\right)+\sum_{\substack{i, j=1 \\ i \neq j}}^{m} b_{i j}\left(n_{i j}-\lambda_{i j} t_{i}\right)+c
$$

for almost all (with respect to $m_{\vartheta_{0}}$ ) points $u \in U$. 
Let

$$
\Lambda_{i}=\left(\lambda_{i 1}, \ldots, \lambda_{i, i-1}, \lambda_{i, i+1}, \ldots, \lambda_{i m}\right) \text {. }
$$

In the sequel we assume that $g$ is a function of only $\Lambda_{i}$ and $m>2$. Without loss of generality let $i=1$.

Two distinct values, $\Lambda_{1}^{(1)}=\left(\lambda_{11}^{(1)}, \ldots, \lambda_{1 m}^{(1)}\right)$ and $\Lambda_{1}^{(2)}=\left(\lambda_{11}^{(2)}, \ldots, \lambda_{1 m}^{(2)}\right)$, are equivalent with respect to $g\left(\Lambda_{1}\right)$ if $g\left(\Lambda_{1}^{(1)}\right)=g\left(\Lambda_{1}^{(2)}\right)$.

THEOREM 3. If for a Markov stopping time $\tau$ there exists a nonconstant estimator $f$ which is efficient for a function $g\left(\Lambda_{1}\right)$ for two values of $\Lambda_{1}$ which are not equivalent with respect to $g\left(\Lambda_{1}\right)$, then there exist constants $\alpha_{1}, \ldots, \alpha_{m}, \beta$, not all zero, and $\gamma \neq 0$ such that

$$
\sum_{j=2}^{m} \alpha_{j} n_{1 j}+\beta t_{1}+\gamma=0 \quad m_{3_{0}} \text {-almost surely. }
$$

TheOrem 4. Let $P\left(T_{1}>0\right)=1$. Let us assume that for a Markov stopping time $\tau$ there exist constants $\alpha_{1}, \ldots, \alpha_{m}, \beta$, not all zero, $\gamma \neq 0$, such that

$$
\sum_{j=1}^{m} \alpha_{j} n_{i j}+\beta t_{1}+\gamma=0 \quad m_{\vartheta_{0}} \text {-almost surely, }
$$

then for almost all $u \in U$ either

$$
n_{1 \sigma(2)}+n_{1 \sigma(3)}+\ldots+n_{1 \sigma(k)}=l
$$

for some positive integer $l$, where $(\sigma(2), \ldots, \sigma(m))$ is a permutation of $(2,3, \ldots, m)$ and $k$ is an integer, $2 \leqslant k \leqslant m$, or

$$
t_{1}=\alpha \quad \text { for some } \quad \alpha>0 \text {. }
$$

Let $\tau$ be the time at which line (2) is first attained for some $k=1,2, \ldots, m$ and some positive integer $l$. Such a plan we shall call an inverse plan.

Let $\tau$ be the time at which the line $t_{1}=\alpha$ is first attained for some $\alpha>0$. Such a plan we shall call a simple plan.

THEOREM 5. The following functions are efficiently estimable ones:

a) for an inverse plan with some $k, l$ and $\sigma$

$$
g\left(\Lambda_{1}\right)=\frac{\alpha_{2} \lambda_{1 \sigma(2)}+\ldots+\alpha_{k} \lambda_{1 \sigma(k)}+\beta}{\lambda_{1 \sigma(2)}+\ldots+\lambda_{1 \sigma(k)}}
$$

b) for a simple plan

$$
g\left(\Lambda_{1}\right)=\alpha_{2} \lambda_{12}+\ldots+\alpha_{m} \lambda_{1 m}+\beta .
$$




\section{Sequential plans for the birth and death process ([5])}

Let $\left(\Omega, \mathscr{F}, P_{\vartheta}\right)$ be a probability space and $\xi(t), t \geqslant 0$, the birth and death process satisfying the following conditions:

$$
\begin{gathered}
P_{\vartheta}(\xi(t+\Delta t)=i+1 \mid \xi(t)=i)=\lambda a_{i} \Delta t+o(\Delta t)>0 \\
P_{\vartheta}(\xi(t+\Delta t)=i-1 \mid \xi(t)=i)=\mu b_{i} \Delta t+o(\Delta t)>0 \\
P_{\Im}(\xi(t+\Delta t)=i \pm k \mid \xi(t)=i)=o(\Delta t)>0, \quad k \geqslant 2, \\
P_{\Im}(\xi(t+\Delta t)=i \mid \xi(t)=i)=1-\left(\lambda a_{i}+\mu b_{i}\right) \Delta t+o(\Delta t)>0 \\
P_{\vartheta}(\xi(0)=n)=1, \\
P_{\vartheta}(\xi(t+\Delta t)<0 \mid \xi(0)=0)=0
\end{gathered}
$$

for every $\vartheta=(\lambda, \mu) \in \Theta \subset(0, \infty) \times(0, \infty)$, where $a_{i}>0$ for every $i \geqslant 0$, and $b_{0}=0, b_{i}>0$ for every $i \geqslant 1$. In the case $a_{0}=0$ we assume that

$$
P_{\vartheta}(\xi(t+\Delta t)>0 \mid \xi(t)=0)=0 \quad \text { for every } \vartheta \in \Theta .
$$

We also assume that almost all realizations of the process have only a finite number of jumps in the interval $[0, t], t \geqslant 0$. The process $\xi(t), t \geqslant 0$, generates the measure $\mu_{9, \mathrm{r}}$ in the space $\left(D_{1}([0, \infty)), \mathscr{Z}_{1, \mathrm{t}}\right)$, where $D_{1}([0, \infty))$ is the space of right continuous, integer-valued functions with unit jumps. The measure $\mu_{\vartheta, t}$ is absolutely continuous with respect to $\mu_{\vartheta_{0}, t}$ and

$$
\frac{d \mu_{9, t}}{d \mu_{9_{0}, t}}=c\left(B(t), D(t), P_{1}(t), P_{2}(t) ; \vartheta_{0}\right) \cdot \lambda^{B(t)} \mu^{D(t)} \exp \left[-\left(\lambda P_{1}(t)+\mu P_{2}(t)\right)\right],
$$

where $B(t)-$ the number of births in the interval $[0, t], D(t)-$ the number of deaths in the interval $[0, t]$,

$$
P_{1}(t)=\sum_{i} a_{i} T_{i}(t), \quad P_{2}(t)=\sum_{i} b_{i} T_{i}(t)
$$

with $T_{i}(t)$ - the total time during which the process remains in the state $i$ in the interval $[0, t]$.

Let us denote

$$
Z(t)=\left(B(t), D(t), P_{1}(t), P_{2}(t)\right) .
$$

By the modification of Sudakov's lemma it follows that the joint distribution $m_{\vartheta}$ of $(\tau, Z(t))$ is absolutely continuous with respect to $m_{\vartheta_{0}}$ and

$$
\begin{aligned}
\frac{d m_{\vartheta}}{d m_{\vartheta_{0}}}(u)=c\left(b(u), d(u), p_{1}(u), p_{2}(u) ; \vartheta_{0}\right) \lambda^{b(u)} \mu^{d(u)} \exp [ & \left.-\left(\lambda p_{1}(u)+\mu p_{2}(u)\right)\right] \\
& =h\left(u ; \vartheta, \vartheta_{0}\right) .
\end{aligned}
$$


THEOREM 6. If $\tau$ is a Markov stopping time, then under the regularity conditions we have

$$
\begin{gathered}
E_{9}\left[\frac{B(\tau)}{\lambda}-P_{1}(\tau)\right]=0, \quad E_{9}\left[\frac{D(\tau)}{\mu}-P_{2}(\tau)\right]=0, \\
E_{\vartheta}\left[\frac{B(\tau)}{\lambda}-P_{1}(\tau)\right]^{2}=\frac{1}{\lambda^{2}} E_{9} B(\tau), \\
E_{\vartheta}\left[\left(\frac{B(\tau)}{\lambda}-P_{1}(\tau)\right)\left(\frac{D(\tau)}{\mu}-P_{2}(\tau)\right)\right]=0
\end{gathered}
$$

and

$$
D_{\vartheta} f(\tau, Z(\tau)) \geqslant \frac{\lambda^{2}}{E_{\vartheta} B(\tau)}\left(\frac{\partial}{\partial \lambda} g(\vartheta)\right)^{2}+\frac{\mu^{2}}{E_{\vartheta} D(\tau)}\left(\frac{\partial}{\partial \mu} g(\vartheta)\right)^{2}
$$

This inequality becomes an equality if and only if

$$
\begin{aligned}
f(u) & =\left(\frac{\partial}{\partial \lambda} g(\vartheta)\right) \frac{\lambda^{2}}{E_{\vartheta} B(\tau)} \frac{\partial}{\partial \lambda} \log h\left(u ; \vartheta, \vartheta_{0}\right)+ \\
& +\left(\frac{\partial}{\partial \mu} g(\vartheta)\right) \frac{\mu^{2}}{E_{\vartheta} D(\tau)} \frac{\partial}{\partial \mu} \log h\left(u ; \vartheta, \vartheta_{0}\right)+g(\vartheta) \quad m_{\vartheta_{0}} \text {-almost surely. }
\end{aligned}
$$

THEOREM 7. If $(\tau, f(\tau, Z(\tau)))$ is an efficient plan for $g(\vartheta)$, then there exist constants $\alpha, \beta, \gamma_{1}, \gamma_{2}, \gamma_{3}$ for which

$$
\alpha b(u)+\beta d(u)+\gamma_{1} p_{1}(u)+\gamma_{2} p_{2}(u)+\gamma_{3}=0 \quad m_{3_{0}} \text {-almost surely, }
$$

with $\alpha^{2}+r_{1}^{2} \neq 0, \beta^{2}+\gamma_{2}^{2} \neq 0$.

We also conclude that only

$$
g(\vartheta)=\frac{k_{0}+k_{1} \lambda \mu+k_{2} \lambda+k_{3} \mu}{l_{0}+l_{1} \lambda \mu+l_{2} \lambda+l_{3} \mu}
$$

is an efficiently estimable function.

EXAMPLES. a) Let $b_{0}=0, a_{i}>0$,

$$
\tau_{t_{0}}(x(\cdot))=\inf \left\{t: \sum_{i} a_{i} T_{i}(t, x(\cdot))=t_{0}\right\} .
$$

Then

$$
E_{\vartheta} P_{1}\left(\tau_{t_{0}}\right)=t_{0} \quad \text { and } \quad D_{\vartheta} P_{1}\left(\tau_{t_{0}}\right)=0
$$

Let $g(\vartheta)=g(\lambda)$. We have

$$
E_{9} B\left(\tau_{t_{0}}\right)=\lambda t_{0} \quad \text { and } \quad D_{9} B\left(\tau_{t_{0}}\right)=\lambda t_{0} .
$$


If the estimator $f\left(\tau_{t_{0}}, Z\left(\tau_{t_{0}}\right)\right)$ is efficient, then it takes the form

$$
f\left(\tau_{t_{0}}, Z\left(\tau_{t_{0}}\right)\right)=c_{1} B\left(\tau_{t_{0}}\right)+c_{2}
$$

and

$$
g(\lambda)=c_{1} \lambda t_{0}+c_{2}
$$

is an efficiently estimable function.

b) Let

$$
\tau_{x_{0}}(x(\cdot))=\inf \left\{t: B(t, x(\cdot))=x_{0}\right\}
$$

Then

$$
E_{\vartheta} B\left(\tau_{x_{0}}\right)=x_{0} \text { and } D_{\vartheta} B\left(\tau_{x_{0}}\right)=0 .
$$

Let $g(\vartheta)=g(\lambda)$. We have

$$
E_{9} P_{1}\left(\tau_{x_{0}}\right)=\frac{x_{0}}{\lambda} \quad \text { and } \quad D_{9} P_{1}\left(\tau_{x_{0}}\right)=\frac{x_{0}}{\lambda^{2}}
$$

So, if the estimator $f\left(\tau_{x_{0}}, Z\left(\tau_{x_{0}}\right)\right)$ is efficient, then there exist constants $c_{1}, c_{2}$ such that

$$
f\left(\tau_{x_{0}}, Z\left(\tau_{x_{0}}\right)\right)=c_{1} P_{1}\left(\tau_{x_{0}}\right)+c_{2}
$$

and

$$
g(\lambda)=c_{1} \frac{x_{0}}{\lambda}+c_{2}
$$

is an efficiently estimable function.

c) Let us take $a_{i}=b_{i}, a_{0}=b_{0}=0$,

$$
\left.\tau_{x_{0}}^{1}(x(\cdot))=\inf _{1}^{\prime} r: B(t, x(\cdot))+D(t, x(\cdot))=x_{0}\right),
$$

where $x_{0}<n$. Then we have

$$
\begin{gathered}
E_{3}\left[B\left(\tau_{x_{0}}^{1}\right)+D\left(\tau_{x_{0}}^{1}\right)\right]=x_{0}, \quad D_{9}\left[B\left(\tau_{x_{0}}^{1}\right)+D\left(\tau_{x_{0}}^{1}\right)\right]=0, \\
P_{1}\left(\tau_{x_{0}}^{1}\right)=P_{2}\left(\tau_{x_{0}}^{1}\right), \\
E_{\vartheta} P_{1}\left(\tau_{x_{0}}^{1}\right)=\frac{1}{\lambda+\mu} x_{0}, \quad E_{\vartheta} B\left(\tau_{x_{0}}^{1}\right)=\frac{\lambda}{\lambda+\mu} x_{0}, \quad E_{\vartheta} D\left(\tau_{x_{0}}^{1}\right)=\frac{\mu}{\lambda+\mu} x_{0}, \\
E_{\vartheta}\left[B\left(\tau_{x_{0}}^{1}\right) D\left(\tau_{x_{0}}^{1}\right)\right]=\frac{\lambda \mu}{(\lambda+\mu)^{2}} x_{0}\left(x_{0}-1\right), \\
D_{9} P_{1}\left(\tau_{x_{0}}^{1}\right)=\frac{1}{(\lambda+\mu)^{2}} x_{0} .
\end{gathered}
$$


Let

$$
f\left(\tau_{x_{0}}^{1}, Z\left(\tau_{x_{0}}^{1}\right)\right)=\frac{1}{x_{0}} P_{1}\left(\tau_{x_{0}}^{1}\right) .
$$

This estimator is efficient for the function

$$
g(\lambda, \mu)=\frac{1}{\lambda+\mu} .
$$

\section{Sequential plans for a Markov process with migration ([3])}

Let us assume that there is a flow of homogeneous objects entering a certain system $A$ and each of the objects in the system may emigrate in one of the $n$ directions $B_{1}, \ldots, B_{n}$. We also assume that the incoming objects form a Poisson flow with intensity $\alpha$. Further, if an object is in the system $A$ at a time $t>0$, then it can emigrate during time $(t, t+\Delta t)$, independently of its entrance time, in the direction $B_{j}, j=1, \ldots, n$, with the probability $\beta_{j} \Delta t$ $+o(\Delta t)$. By $V(t)$ let us denote the number of objects which entered the system during the time interval $[0, t)$. Let $W_{j}(t)$ be the number of objects which emigrated during this time in the direction $B_{j}, j=1,2, \ldots, n$, and let $k_{0}$ be the number of objects present in $A$ at the time $t=0$. Let $I=\{0,1,2, \ldots\}$ and $T=[0, \infty)$. Next, let us denote $W_{0}(t)=k_{0}+V(t)$ and let $\vartheta$ be the vector $\left(\alpha, \beta_{1}, \ldots, \beta_{n}\right) \in \Theta \subset(0, \infty)^{n+1}$

Let $\left(\Omega, \overline{\mathscr{F}}, P_{\vartheta}\right)$ be a probability space. Let us consider a homogeneous Markov process

$$
\xi(t)=\left(W_{0}(t), W_{1}(t), \ldots, W_{n}(t)\right), \quad t \in T,
$$

defined on $\left(\Omega, \mathscr{F}, P_{\Im}\right)$. The values of this process we shall denote by $x$ $=\left(w_{0}, w_{1}, \ldots, w_{n}\right)$.

We assume that the process $\xi(t), t \in T$, satisfies the following conditions:

a) $P_{s}\left(\xi(0)=\left(k_{0}, 0, \ldots, 0\right)\right)=1$,

b) the transition probabilities are of the form

$P_{s}(\xi(t+\Delta t)=y \mid \xi(t)=x)$

$=\left\{\begin{array}{l}\alpha \Delta t+o(\Delta t) \\ \sum_{j=1}^{n} k \beta_{j} \Delta t+o(\Delta t) \\ 1-\left(\alpha+\sum_{j=1}^{n} k \beta_{j}\right) \Delta t+o(\Delta t) \\ o(\Delta t)\end{array}\right.$

$$
\text { if } \begin{aligned}
& x=\left(w_{0}, w_{1}, \ldots, w_{n}\right) \text { and } \\
& y=\left(w_{0}+1, w_{1}, \ldots, w_{n}\right), \\
& \text { if } \quad \begin{aligned}
x & =\left(w_{0}, w_{1}, \ldots, w_{n}\right) \text { and } \\
y & =\left(w_{0}, w_{1}, \ldots, w_{j-1}, w_{j}+1, w_{j+1}, \ldots, w_{n}\right), \\
j & =1, \ldots, n,
\end{aligned} \\
& \text { if } \quad x=y, \\
& \text { otherwise, }
\end{aligned}
$$


where $k=w_{0}-\sum_{j=1}^{n} w_{j}$ denotes the value of the random variable,

$$
K(t)=W_{0}(t)-\sum_{j=1}^{n} W_{j}(t)
$$

determining the number of objects present in the system at time $t$,

c) $P_{s}(K(t) \geqslant 0)=1$ for every $t>0$.

Our problem is to estimate the intensities $\alpha, \beta_{1}, \ldots, \beta_{n}$ or their functions, using the observation of the process $\xi(t), t \in T$. From the Skorokhod theorems ([1]) we have $\mu_{9, t} \ll \mu_{9_{0}, t}$ and

$$
\begin{aligned}
\frac{d \mu_{9, t}}{d \mu_{3_{0}, t}}(x(\cdot))=\left(\frac{\alpha}{\alpha_{0}}\right)^{v(t)} \exp & {\left[-\left(\alpha-\alpha_{0}\right) t-\sum_{j=1}^{n}\left(\beta_{j}-\beta_{0 j}\right)\right.} \\
& \left.\times\left(k(t) t+\sum_{j=1}^{n} \sum_{t=1}^{w_{j}(t)} \sigma_{j l}-\sum_{r=1}^{v(t)} v_{r}\right)\right] \prod_{j=1}^{n}\left(\frac{\beta_{j}}{\beta_{0 j}}\right)^{w_{j}(t)},
\end{aligned}
$$

where $v_{r}$ 's are the arrival times,

$$
0<v_{1}<\ldots<v_{v(t)}<t
$$

and $\sigma_{j l}$ 's are the exit times in direction $B_{j}$,

$$
0<\sigma_{j 1}<\ldots<\sigma_{j w_{j}(t)}<t, \quad j=1, \ldots, n .
$$

Let us denote

$$
\begin{gathered}
w(t)=\left(w_{1}(t), \ldots, w_{n}(t)\right) \\
S(t, x(\cdot))=k(t) t+\sum_{j=1}^{n} \sum_{t=1}^{w_{j}(t)} \sigma_{j l}-\sum_{r=1}^{v(t)} v_{r} \\
\beta=\sum_{j=1}^{n} \beta_{j}, \quad \beta_{0}=\sum_{j=1}^{n} \beta_{0 j}
\end{gathered}
$$

and

$$
Z(t, x(\cdot))=(v(t), w(t), S(t, x(\cdot)))
$$

The function $S$ determines the overall time spent in the system by the objects which arrived during the time $[0, t)$ or were in the system at the time $t=0$. So we can write

$$
\begin{aligned}
\frac{d \mu_{S_{, t}}}{d \mu_{\vartheta_{0}, t}}(x(\cdot)) & =\left(\frac{\alpha}{\alpha_{0}}\right)^{v(t)} \exp \left[-\left(\alpha-\alpha_{0}\right) t-\left(\beta-\beta_{0}\right) S(t, x(\cdot))\right] \prod_{j=1}^{n}\left(\frac{\beta_{j}}{\beta_{0_{j}}}\right)^{w_{j}(t)} \\
& =c\left(t, Z(t, x(\cdot)) ; \vartheta_{0}\right) \cdot \alpha^{v(t)} \exp [-\alpha t-\beta S(t, x(\cdot))] \prod_{j=1}^{n} \beta_{j}^{w_{j}(t)}
\end{aligned}
$$


From the modification of Sudakov's lemma we have

$$
\frac{d m_{\vartheta}}{d m_{s_{0}}}(u)=c\left(u ; \vartheta_{0}\right) \alpha^{v(u)} \exp [-\alpha t(u)-\beta s(u)] \prod_{j=1}^{n} \beta_{j}^{w_{j}(u)} .
$$

The Cramér-Rao inequality takes the form

$$
D_{\vartheta} f(\tau, Z(\tau)) \geqslant \frac{\alpha}{E_{\vartheta} \tau}\left[g_{\alpha}^{\prime}(\vartheta)\right]^{2}+\frac{1}{E_{\vartheta} S(\tau)} \sum_{j=1}^{n} \beta_{j}\left[g_{j}^{\prime}(\vartheta)\right]^{2} .
$$

The equality holds at a particular value of $\vartheta$ iff

$$
f(\tau, Z(\tau))=\frac{g_{\alpha}^{\prime}(\vartheta)}{E_{\vartheta} \tau}[V(\tau)-\alpha \tau]+\frac{1}{E_{\vartheta} S(\tau)} \sum_{j=1}^{n} g_{j}^{\prime}(\vartheta)\left[W_{j}(\tau)-\beta_{j} S(\tau)\right]+g(\vartheta) .
$$

EXAMPLES, a) Let

$$
\tau^{(1)}(x(\cdot))=T_{0}>0 .
$$

For this stopping time

$$
f^{(1)}=\frac{c_{1}}{T_{0}} V\left(T_{0}\right)+c_{2}
$$

is efficient for $g(\vartheta)=c_{1} \alpha+c_{2}$.

b)

$$
\tau^{(2)}(x(\cdot))=\inf \left\{t: v(t)=v_{0}\right\},
$$

where $v_{0}$ is a positive integer number.

For this stopping time

$$
f^{(2)}=\frac{c_{1}}{v_{0}} \tau^{(2)}+c_{2}
$$

is efficient for $g(\vartheta)=c_{1} / \alpha+c_{2}$.

c)

$$
\tau^{(3)}(x(\cdot))=\inf \left\{t: S(t, x(\cdot))=s_{0}\right\}, \quad s_{0}>0 .
$$

For this stopping time

$$
f^{(3)}=\frac{1}{s_{0}} \sum_{j=1}^{n} c_{j} W_{j}\left(\tau^{(3)}\right)+d
$$

is efficient for $g(9)=\sum_{j=1}^{n} c_{j} \beta_{j}+d$.

d)

$$
\tau^{(4)}(x(\cdot))=\inf \left\{t: \sum_{i=1}^{k} w_{\sigma(i)}(t)=m_{0}\right\}
$$


where $m_{0}$ is a positive integer number, $2 \leqslant k \leqslant n, \sigma$ is a permutation of the set $\{1,2, \ldots, n\}$. For this stopping time

$$
f^{(4)}=\frac{1}{m_{0}} \sum_{i=1}^{k} c_{i} W_{\sigma(i)}\left(\tau^{(4)}\right)+d
$$

is efficient for

$$
g(\vartheta)=\left(\sum_{i=1}^{k} c_{i} \beta_{\sigma(i)}\right)\left(\sum_{i=1}^{k} \beta_{\sigma(i)}\right)^{-1}+d
$$

e)

$$
\tau^{(5)}(x(\cdot))=\inf \left\{t: w_{j}(t)=l_{0}\right\}
$$

where $t_{0}$ is positive integer.

For this stopping time

$$
f^{(5)}=\frac{c_{1}}{l_{0}} S\left(\tau^{(5)}\right)+c_{2}
$$

is efficient for $g(\vartheta)=c_{1} / \beta_{j}+c_{2}$.

\section{References}

[1] И.И. Гихман, А.В. Скороход, О пнотностях вероятиостиых иср в фуикциона.Іыных nрocmpancmeax. Успехи математических наук 21 (1966). 8.3-152.

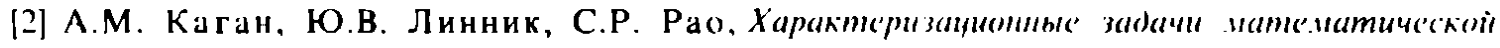
(momumuки. Наука. Москва 1972.

[3] R. Magiera, Sequential estimation of the transition intensities in Markov processes with migration, Zastos. Mat. 18 (1984), 241-250.

[4] R. Różański, A modification of Sudakov's lemma and efficient sequential plans for the Ornstein-Uhlenbeck process, Zastos. Mat. 17 (1980), 73-86.

[5] -, Charakteryzacja efektywnych planów sekwencyjnych dla procesu urodzin i śmierci, Mat. Stosowana, 17 (1980), 83-93.

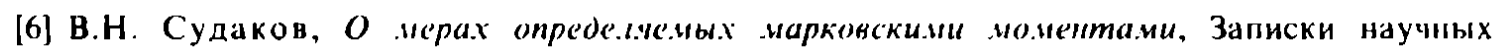
семинаров Л.О.М.И.. Том 12, Исслслования по теории с.Іучайыњх пролессов. 1969. $157-164$.

[7] S. Trybula, Sequential estimation in proc'esses with independent increments, Dissertationes Math. (Rozprawy Mat.) 60 (1968).

[8] -, Sequential estimation for finite state Markov processes, Zastos. Mat. 17 (1982), 227-248.

Presented to the semester

Sequential Methods in Statistics

September 7-December 11, 1981 